\title{
Agent independent probabilistic robustness certificates for robust optimization programs with uncertain quadratic cost
}

\author{
George Pantazis, Filiberto Fele and Kostas Margellos
}

\begin{abstract}
This paper focuses on a specific class of convex multi-agent programs, prevalent in many practical applications, where agents cooperate to minimize a common cost, expressed as a function of the aggregate decision and affected by uncertainty. We model uncertainty by means of scenarios and use an epigraphic reformulation to transfer the uncertain part of the cost function to the constraints. Then, by exploiting the structure of the program under study and leveraging on existing results in the scenario approach literature, and in particular using the so called support rank notion, we provide for the optimal solution of the program distributionfree robustness certificates that are agent-independent, i.e., the constructed bound on the probability of constraint violation does not depend on the number of agents, but only on the dimension of the agents' decision. This leads to a significant improvement as it substantially reduces the number of samples required to achieve a certain level of probabilistic robustness as the number of agents increases. Our certificates can be used alongside any convex optimization algorithm centralised, decentralised or distributed, to obtain an optimal solution of the underlying problem. Our theoretical results are accompanied by a numerical example that investigates the electric vehicle charging problem and validates that the obtained robustness certificate is independent of the number of vehicles in the fleet.
\end{abstract}

\section{INTRODUCTION}

\section{A. Motivation}

A vast amount of today's challenges in the domains of energy systems [1], [2], traffic networks [3], economics [4] and the social sciences [5], [6] revolve around multiagent systems, i.e., systems which comprise different entities/agents that interact with each other and make decisions, based on individual or collective criteria. Existing literature provides a plethora of methods to solve such problems. Each method is appropriately designed to fit the structure of these interactions and the agents' incentives. To address challenges, related to the computational complexity issues and privacy concerns of solving a multi-agent optimization problem in a centralised fashion, several decentralised or distributed coordination schemes have been proposed [7], [8]. In the decentralised case, agents optimize their cost function locally and then communicate their strategies to a central authority that is responsible for the information they exchange. In the distributed case, a central authority is absent and agents communicate via a connected network usually with their local objective functions and local decision

Research was supported by the UK Engineering and Physical Sciences Research Council (EPSRC) under grant agreement EP/P03277X/1.The authors are with the Department of Engineering Science, University of Oxford, OX1 3PJ, UK, georgios.pantazis@Imh.ox.ac.uk, filiberto.fele@eng.ox.ac.uk, kostas.margellos@eng.ox.ac.uk sets only known to them. In most practical applications, the presence of uncertainty in such problems constitutes a critical factor that, if not taken into account, could lead to unpredictable behaviour. As such, it is of major importance to accompany the solutions of such algorithms with robustness certificates that can mitigate the effects of uncertainty.

In this paper we focus on a specific class of multiagent optimization programs, where the agents' strategies are subject to constraints separable among agents and coupled via a common convex cost that is a function of the aggregate decision. This formulation has been considered in [9] and is extended to our set-up to account for the case, in which the cost function is affected by uncertainty. As in [9] the constraints of each agent are of deterministic nature. Problems whose structure shares similarities with ours have been considered in [10], [11], [12], though under a purely deterministic and competitive set-up. In our case, the sample set and probability distribution of the uncertain parameter are considered unknown, which urges us to adopt a data-driven approach, where the uncertainty is represented by means of scenarios that could be either available as historical data, or extracted via some prediction model. Working under the theoretical framework of the so called scenario approach [13], [14], [15] and based on the results of [16], we aim at improving the probabilistic guarantees that the optimal value of the cost will not escalate after the occurrence of a new yet unseen uncertainty realisation. This is achieved by transferring the uncertain part of the cost function to the constraints, using an epigraphic reformulation and providing feasibility probabilistic guarantees for the optimal solution of this equivalent problem. Our choice for using the scenario approach instead of other available techniques lies in the fact that it is a distribtion-free methodology, i.e., it requires no knowledge of the uncertainty set or the probability distribution, unlike robust [17] and stochastic optimization [18], where this information is necessary.

In [19] it is shown that the number of support constraints/ support samples (at the core of the scenario approach theory) of a convex optimization problem, can in turn be bounded by the dimension of the optimization variable. This fact is then used to provide probabilistic feasibility guarantees for the optimal solution in an a priori fashion. In [16] a tighter bound is offered exploiting the property that, in many cases of practical interest, a random constraint may leave a subspace of the decision space unconstrained for all uncertainty realizations. As such, [16] introduces the concept of the support rank, obtained by removing from the problem's dimension the dimension of the maximal 
unconstrained subspace.

\section{B. Contributions}

The main contributions of this paper are as follows:

1) Leveraging the results in [16], we construct an upper bound for the number of support samples shown to be agent independent, i.e., it only depends on the dimension $n$ of agents' decision vectors and does not change as the number of agents $N$ increases. Specifically, we show that the support rank of the random constraint can be upper bounded by the quantity $n+1$. This refined bound constitutes a major improvement for this class of problems, when compared with the bound of the problem's dimension $n N+1$ provided by the classic results in the scenario approach [19].

2) An immediate consequence of our main result is that the number of samples required to provide identical probabilistic guarantees with the classic scenario theory, is substantially less as the number of agents increases. This is of crucial importance in large-scale applications, where a large number of agents is present.

A similar claim that agent independent bounds could be derived for a class of games with structure similar to that of our optimization problem, was conjectured in [20], [21]. Therein, by adopting a variational inequalities approach, the authors provide probabilistic guarantees for the Nash equilibria of a game affected by uncertainty. The developed a priori bound, is agent dependent and equal to the dimension of the problem under study $n N+1$. To the best of our knowledge, this is the first time that agent independent robustness certificates are provided for optimization programs of this class.

The rest of the paper is organized as follows: Section II introduces the problem under study and presents our motivating example, i.e., the electric vehicle charging control problem. Section III contains the theoretical analysis and proof of the main results, namely, providing agent independent distribution-free probabilistic guarantees for the class of problems under study. Section IV provides a numerical study for the electric vehicle application introduced in Section II. Finally, Section V concludes the paper and provides some potential future research directions.

\section{Problem Statement}

\section{A. Robust optimization programs with uncertain cost}

Let $\mathscr{N}=\{1, \ldots, N\}$ be the index set of all agents, where $N$ denotes their total number and $x_{i}$ the strategy of agent $i$ taking values in the deterministic set $X_{i} \subseteq \mathbb{R}^{n}$. We denote $x=\left(x_{i}\right)_{i \in \mathscr{N}} \in X=\prod_{i=1}^{N} X_{i} \subseteq \mathbb{R}^{n N}$ the collection of all agents' strategies. The cost function is affected by an uncertainty parameter $\theta$ defined on the (possibly unknown) probability space $(\Theta, \mathscr{F}, \mathbb{P})$, where $\Theta$ is the sample space, equipped with a $\sigma$-algebra $\mathscr{F}$ and a probability measure $\mathbb{P}$. The vector $x_{-i}=$ $\left(x_{j}\right)_{j \in \mathscr{N}, j \neq i} \in \prod_{j \in \mathscr{N}, j \neq i} X_{j} \subseteq \mathbb{R}^{n(N-1)}$ denotes the collection of the decision vectors of all other agents' strategies except for that of agent $i$. The symbols $x$ and $\left(x_{i}, x_{-i}\right)$ are used interchangeably in this paper, depending on the context. Finally, define $\left\{\theta_{m}\right\}_{m \in \mathscr{M}} \in \Theta^{M}$, where $\mathscr{M}=\{1, \ldots, M\}$ as a finite collection of $M$ independent and identically distributed (i.i.d.) scenarios/realisations of the uncertain vector $\theta$, where $\Theta^{M}$ is the cartesian product of multiple copies of the sample space $\Theta$.

We consider the following program:

$$
P: \min _{\left\{x_{i} \in X_{i}\right\}_{i \in \mathscr{N}}} J(x),
$$

where $J(x)=f(x)+\max _{\theta \in \Theta} g(x, \theta)$ and $f: X \rightarrow \mathbb{R}, g: X \times \Theta \rightarrow$ $\mathbb{R}$ is the deterministic and the uncertain part of the cost function, respectively. In addition, we impose the following standing assumption

Assumption 1: 1) The deterministic part $f$ is jointly convex with respect to all agents' decision vectors, and the sets $X_{i}$ are nonempty, convex and compact for any $i \in \mathscr{N}$.

2) The uncertain part $g$ takes the aggregative form

$$
\begin{array}{r}
g(x, \theta)=\sum_{i \in \mathscr{N}} g_{i}\left(\left(x_{i}, x_{-i}, \theta\right),\right. \text { where } \\
g_{i}\left(x_{i}, x_{-i}, \theta\right)=x_{i}^{T}(A(\theta) \sigma(x)+b(\theta)) .
\end{array}
$$

Note that $\sigma: X \rightarrow \mathbb{R}^{n}$ is a mapping $\left(x_{i}\right)_{i \in \mathscr{N}} \mapsto \sum_{i \in \mathscr{N}} x_{i}$ and $A: \Theta \rightarrow \mathbb{R}^{n \times n}, b: \Theta \rightarrow \mathbb{R}^{n}$ are uncertain mappings with $A(\theta) \succcurlyeq 0$ and $A(\theta)=A^{T}(\theta)$ for all $\theta \in \Theta$.

Under Assumption 1 the function $J$ is convex, as the pointwise maximum of a countable number of convex functions is itself a convex function [22]. From Assumption 1.2 the uncertain counterpart of the cost function under study takes the form

$$
g(x, \theta)=\sigma(x)^{T}(A(\theta) \sigma(x)+b(\theta)) .
$$

Since $g$ is convex, using an epigraphic reformulation we recast $P$ to the equivalent semi-infinite program

$$
\begin{aligned}
P^{\prime} & : \min _{\left\{x_{i} \in X_{i}\right\}_{i \in \mathscr{N}}, \gamma \in \mathbb{R}} f(x)+\gamma \\
& \text { subject to } h(x, \gamma, \theta) \leq 0, \forall \theta \in \Theta,
\end{aligned}
$$

where $h(x, \gamma, \theta)=g(x, \theta)-\gamma$. Note that lifting the dimension of the problem by one by introducing the epigraphic variable $\gamma$, allows moving the uncertain part from the cost function to the constraints. In addition, if $\left(x^{*}, \gamma^{*}\right)$ is the optimal solution of problem $P^{\prime}$, then $x^{*}$ is the optimal solution of the original problem $P$. Due to the presence of uncertainty and the (possibly) infinite cardinality of $\Theta$, problem $P^{\prime}$ is very difficult to solve, without imposing any further assumptions on the geometry of the sample set $\Theta$ and/or the underlying probability distribution $\mathbb{P}$. To overcome this issue, we adopt the scenario approach [23], which involves drawing a finite number of samples from the sample set $\Theta$ and forming an optimization program subject to a finite amount of constraints. This program, known in the scenario approach literature as a scenario program, constitutes an approximation to the original semi-infinite program $P^{\prime}$. In addition, it circumvents the challenges of its semi-infinite 
counterpart. The corresponding scenario program of the uncertain semi-infinite program $P^{\prime}$ is thus given by

$$
\begin{aligned}
P_{S C}^{\prime} & \min _{\left\{x_{i} \in X_{i}\right\}_{i \in \mathscr{N}}, \gamma \in \mathbb{R}} f(x)+\gamma \\
& \text { subject to } h\left(x, \gamma, \theta_{m}\right) \leq 0, \forall m \in \mathscr{M},
\end{aligned}
$$

where $\left\{\theta_{m}\right\}_{m \in \mathscr{M}} \in \Theta^{M}$ are i.i.d. multi-samples of cardinality M.

The proposed structure captures a wide class of engineering problems. A notable application that constitutes our motivating example is the electric vehicle (EV) charging problem detailed in the next subsection.

\section{B. Example: Electric vehicle charging control}

Let $x_{i}=\left(x_{i}^{(t)}\right)_{t=1}^{n}$ denote the charging schedule of each electric vehicle $i$ over a charging period of duration $n$. The electricity price is considered to be a random variable affected by uncertainty. All electric vehicles cooperate with each other choosing their charging schedules so as to minimize the total electricity cost, while satisfying certain imposed constraints. Taking all these aspects into account, we consider the following uncertain electric vehicle charging problem

$$
\begin{aligned}
P_{E V}: & \min _{x \in \mathbb{R}^{n N}} f(x)+\max _{\theta \in \Theta} g(x, \theta), \\
& \text { subject to } x_{i} \in\left[\underline{x}_{i}, \bar{x}_{i}\right], \sum_{t=1}^{n} x_{i}^{(n)} \geq E_{i}, \text { for all } i \in \mathscr{N},
\end{aligned}
$$

where $f(x)=\sum_{i \in \mathscr{N}} f_{i}\left(x_{i}, x_{-i}\right)=\sigma(x)^{T} p_{0}(\sigma(x))$ is the deterministic part of the electricity cost that depends on a nominal electricity price $p_{0}(\sigma(x))=A_{0} \sigma(x)+b_{0}$ that is, in turn, a function of the aggregate consumption of the vehicles. Respectively, $g(x, \theta)=\sum_{i \in \mathscr{N}} g_{i}\left(\left(x_{i}, x_{-i}, \theta\right)=\sigma(x)^{T} p(\sigma(x), \theta)\right.$ constitutes the uncertain part of the electricity cost, where the price $p(\sigma(x), \theta)=A(\theta) \sigma(x)+b(\theta)$ is additionally affected by the uncertain parameter $\theta$ extracted from the support set $\Theta$ according to a probability distribution $\mathbb{P}$, where $\Theta$ and $\mathbb{P}$ are considered unknown. Both $A_{0}, A(\theta) \in \mathbb{R}^{n \times n}$ are symmetric positive semi-definite matrices and $b_{0}, b(\theta) \in \mathbb{R}^{n}$ for any $\theta \in \Theta$. The vectors $\underline{x}_{i}, \bar{x}_{i} \in \mathbb{R}^{n}$ constitute the lower and upper bound of the charging rate of vehicle $i \in \mathscr{N}$, respectively, while $E_{i} \in \mathbb{R}$ is the final energy to be achieved by each vehicle $i \in \mathscr{N}$ by the end of the charging cycle.

Following the same lines as in Section II-A, we apply an epigraphic reformulation to obtain the following equivalent semi-infinite program

$$
\begin{gathered}
P_{E V}^{\prime}: \min _{(x, \gamma) \in \mathbb{R}^{n N+1}} f(x)+\gamma, \\
\text { subject to } x_{i} \in\left[\underline{x}_{i}, \bar{x}_{i}\right], \sum_{t=1}^{n} x_{i}^{(n)} \geq E_{i} \text { for all } i \in \mathscr{N}, \\
g(x, \theta) \leq \gamma \text { for all } \theta \in \Theta .
\end{gathered}
$$

Since electricity price volatility is an outcome of a variety of unpredictable factors, such as the cost of fuels, the operating cost of power plants, the maintenance of transmission and distribution networks and the weather conditions, it is very difficult to address the problem using traditional probabilistic approaches. We thus adopt a scenario-based scheme. For the convenience of the reader, some basic concepts related to the scenario approach are introduced in the next subsection, appropriately adapted to fit in our context.

\section{AGENT INDEPENDENT PROBABILISTIC FEASIBILITY GUARANTEES}

\section{A. Basic concepts in the scenario approach}

The concept of the support sample and support constraint is at the core of the scenario approach and is defined as follows.

Definition 1: (Support Sample/ Support Constraint [19]) Fix any i.i.d. multisample $\left\{\theta_{m}\right\}_{m \in \mathscr{M}} \in \Theta^{M}$ and let $x_{0}^{*}=$ $x_{0}^{*}\left(\left\{\theta_{m}\right\}_{m \in \mathscr{M}}\right)$ be the unique optimal solution of the scenario program, when all the $M$ samples are taken into account. Let $x_{-s}^{*}=x_{-s}^{*}\left(\left\{\theta_{m}\right\}_{m \in \mathscr{M}} \backslash \theta_{s}\right)$ be the optimal solution obtained after removal of sample $\theta_{s}$. Then, if $x_{0}^{*} \neq x_{-s}^{*}$ we say that the sample $\theta_{s}$ is a support sample. The constraint that corresponds to the support sample is called a support constraint.

We introduce the following assumption:

\section{Assumption 2: (Adapted from [16])}

1) For each multi-sample $\left\{\theta_{m}\right\}_{m \in \mathscr{M}}$, the scenario program $P_{S C}^{\prime}$ admits a feasible solution.

2) The optimal solution $\left(x^{*}, \gamma^{*}\right)$ of the scenario program $P_{S C}^{\prime}$ is unique.

Note that in case multiple optimal points exist, one can use a convex tie-break rule to select a unique solution.

In many practical applications there are cases where a random constraint may leave a linear subspace unconstrained for any possible sample $\theta \in \Theta$. This observation motivated the concept of the support rank as introduced in [16]. The notion of the support rank is presented formally in Definition 2. Let $y \in \mathbb{Y} \subseteq \mathbb{R}^{d}$, where $d$ is the dimension of the decision vector $y$. Consider the following semi-infinite optimization program

$$
\begin{aligned}
& \min _{y \in \mathbb{Y}} c^{T} y \\
& \text { subject to } l(y, \theta) \leq 0, \forall \theta \in \Theta .
\end{aligned}
$$

Denoting the collection of all the linear subspaces as $\mathscr{L}$, we consider all the linear subspaces $L \in \mathscr{L}$ that, under the presence of the random constraint (6), remain unconstrained for any uncertainty realization $\theta \in \Theta$ and any point $y \in \mathbb{Y}$, i.e., the set

$$
\begin{aligned}
& \mathscr{U}=\bigcap_{\theta \in \Theta} \bigcap_{y \in \mathbb{Y}}\{L \in \mathscr{L}: L \subset F(y, \theta)\}, \\
& \text { where } F(y, \theta)=\left\{\xi \in \mathbb{R}^{d}: l(y+\xi, \theta)=l(y, \theta)\right\} .
\end{aligned}
$$

Definition 2: (Support rank [16])

The support rank $\rho \in\{0, \ldots, d\}$ of a random constraint equals to the dimension of the problem $d$ minus the dimension of the maximal ${ }^{1}$ unconstrained linear subspace $L_{\text {max }}$, i.e, $\rho=d-\operatorname{dim}\left(L_{\max }\right)$.

\footnotetext{
${ }^{1}$ By maximal unconstrained subspace we mean the unique maximal element $L_{\text {max }} \in \mathscr{U}$ for which $L \subseteq L_{\max }$, for all $L \in \mathscr{U}$.
} 
From the support rank lemma (see Lemma 3.8 in [16]) we have that Helly's dimension can be upper bounded by the support rank instead of the dimension $d$ of the problem, which is a more conservative but useful upper bound [19], i.e, $\zeta \leq \rho \in\{0, \ldots, d\}$. Keeping this relation in mind, our main goal is to obtain a bound for the support rank for the problem $P^{\prime}$, thus improving the robustness certificates of its optimal solution.

\section{B. Bounding the support rank}

The following proposition, which together with Theorem 1 constitute the main results of this paper, aims at finding an upper bound for the support rank $\rho$ of the random constraint (2), that is independent from the number of agents involved in the optimization program.

Proposition 1: Under Assumption 1, the support rank $\rho$ of the random constraint (2) in $P^{\prime}$, has an agent-independent upper bound, and in particular, $\rho \leq n+1$.

Proof: The dimension of the problem under study is $d=n N+1$, due to the presence of the epigraphic variable. Let $\mathscr{L}$ be the collection of all linear subspaces in $\mathbb{R}^{n N+1}$. We aim at finding the dimension of the subspace that remains unconstrained for a scenario program subject to the random constraint $h(x, \gamma, \theta) \leq 0$ for any uncertain realisation $\theta \in \Theta$ and any decision vector $(x, \gamma) \in X \times \mathbb{R}$. We first define the collection of linear subspaces that are contained in all the sets $F(x, \gamma, \theta)$ :

$$
\begin{gathered}
\mathscr{U}=\bigcap_{\theta \in \Theta} \bigcap_{(x, \gamma) \in \mathbb{R}^{n N+1}}\{L \in \mathscr{L}: L \subset F(x, \gamma, \theta)\}, \text { where } \\
F(x, \gamma, \theta)=\left\{\left(\xi, \xi^{\prime}\right) \in \mathbb{R}^{n N+1}:\right. \\
\\
\left.h\left(x+\xi, \gamma+\xi^{\prime}, \theta\right)=h(x, \gamma, \theta)\right\}
\end{gathered}
$$

In our case, $h\left(x+\xi, \gamma+\xi^{\prime}, \theta\right)=h(x, \gamma, \theta)$ yields:

$$
\begin{aligned}
& \sigma(x+\xi)^{T}(A(\theta) \sigma(x+\xi)+b(\theta))-\left(\gamma+\xi^{\prime}\right)= \\
= & \sigma(x)^{T}(A(\theta) \sigma(x)+b(\theta))-\gamma, \\
\Longleftrightarrow & \sigma^{T}(x) A(\theta) \sigma(\xi)+\sigma^{T}(\xi) A(\theta) \sigma(x)+ \\
& \sigma^{T}(\xi) A(\theta) \sigma(\xi)+\sigma^{T}(\xi) b(\theta)-\xi^{\prime}=0, \\
\Longleftrightarrow & \sigma^{T}(\xi) A^{T}(\theta) \sigma(x)+\sigma^{T}(\xi)(A(\theta) \sigma(x)+b(\theta))+ \\
& \sigma^{T}(\xi) A(\theta) \sigma(\xi)-\xi^{\prime}=0,
\end{aligned}
$$

where the first equivalence stems from the fact that $\sigma(x+$ $\xi)$ is linear with respect to its arguments, and the second one after some algebraic rearrangement. Note that each of the terms above is scalar, which means that it is equal to its transpose $\forall x \in X$ and $\theta \in \Theta$, while by Assumption 1, $A^{T}(\theta)=A(\theta)$ for any uncertainty realization $\theta \in \Theta$. As such,

$$
\sigma^{T}(\xi)(2 A(\theta) \sigma(x)+b(\theta))+\sigma^{T}(\xi) A(\theta) \sigma(\xi)-\xi^{\prime}=0 .
$$

Using the equalities $\sigma^{T}(\xi)(2 A(\theta) \sigma(x)+b(\theta))=\left(\mathbb{1}_{1 \times N} \otimes\right.$ $\left.(2 A(\theta) \sigma(x)+b(\theta))^{T}\right) \xi$ and $\sigma^{T}(\xi) A(\theta) \sigma(\xi)=\xi^{T}\left(\mathbb{1}_{N \times N} \otimes\right.$ $A(\theta)) \xi$, where $\mathbb{1}_{1 \times N}$ denotes a row vector with all elements being equal to one and $\otimes$ denotes the Kronecker product, (7) can be written in the following form:

$$
\begin{aligned}
& \left(\mathbb{1}_{1 \times N} \otimes\left(2 \sigma^{T}(x) A(\theta)+b^{T}(\theta)\right)\right) \xi+ \\
& \xi^{T}\left(\mathbb{1}_{N \times N} \otimes A(\theta)\right) \xi-\xi^{\prime}=0,
\end{aligned}
$$

Let $\tilde{C}: X \times \Theta \rightarrow \mathbb{R}^{n N}, \tilde{A}: \Theta \rightarrow \mathbb{R}^{n N \times n N} \tilde{C}(x, \theta)=\mathbb{1}_{1 \times N} \otimes$ $\left(2 \sigma^{T}(x) A(\theta)+b^{T}(\theta)\right)$ and $\tilde{A}(\theta)=\mathbb{1}_{N \times N} \otimes A(\theta)$, respectively. Then, equation (8) can be written as:

$$
\begin{aligned}
& \tilde{C}(x, \theta) \xi+\xi^{T} \tilde{A}(\theta) \xi-\xi^{\prime}=0, \\
\Longleftrightarrow & \left(\begin{array}{ll}
\tilde{C}(x, \theta) & -1
\end{array}\right)\left(\begin{array}{c}
\xi \\
\xi^{\prime}
\end{array}\right) \\
& +\left(\begin{array}{ll}
\xi & \xi^{\prime}
\end{array}\right)\left(\begin{array}{cc}
\tilde{A}(\theta) & 0_{n N \times 1} \\
0_{1 \times n N} & 0
\end{array}\right)\left(\begin{array}{l}
\xi \\
\xi^{\prime}
\end{array}\right)=0, \\
\Longleftrightarrow & V(x, \theta) w+w^{T} P(\theta) w=0,
\end{aligned}
$$

where, $V(x, \theta)=\left(\begin{array}{ll}\tilde{C}(x, \theta) & -1\end{array}\right), P(\theta)=\left(\begin{array}{cc}\tilde{A}(\theta) & 0_{n N \times 1} \\ 0_{1 \times n N} & 0\end{array}\right)$ and $w=\left(\begin{array}{c}\xi \\ \xi^{\prime}\end{array}\right)$. We need to find an unconstrained linear subspace that is a subset of $F(x, \gamma, \theta)$. As such, we define

$$
L(x, \gamma, \theta)=\left\{w \in \mathbb{R}^{n N+1}:\left(\begin{array}{c}
P(\theta) \\
V(x, \theta)
\end{array}\right) w=0\right\} .
$$

We can easily see that $L(x, \gamma, \theta) \subset F(x, \gamma, \theta)$. As such, the random constraint $h(x, \gamma, \theta) \leq 0$ cannot constrain any of the dimensions of $L(x, \gamma, \theta)$, which for simplicity we also denote as $L$. Let $Q(x, \gamma, \theta)=\left(\begin{array}{c}P(\theta) \\ V(x, \theta)\end{array}\right)$. Then $L(x, \gamma, \theta)=$ nullspace $(Q(x, \gamma, \theta))$ and from nullity-rank theorem we have that $\operatorname{dim}(L(x, \gamma, \theta))=n N+1-\operatorname{rank}(Q(x, \gamma, \theta))$. Since $\operatorname{rank}(P(\theta))=n$ and $\operatorname{rank}(V(x, \theta))=1$, this means that $\operatorname{rank}(Q(x, \gamma, \theta))=n+1$, which implies that $\operatorname{dim}(L(x, \gamma, \theta))=$ $n N+1-(n+1)$. Notice that the unconstrained subspace that we chose may not be the maximal one, i.e., the linear subspace $L_{\max } \in \mathscr{U}$ for which $L \subseteq L_{\max }$ for any $L \in \mathscr{U}$ (see Definition 2 for a formal definition of $L_{\max }$ ). This means that the support dimension is $\rho=n N+1-\operatorname{dim}\left(L_{\text {max }}\right) \leq$ $n N+1-\operatorname{dim}(L)=n+1$.

An immediate consequence of Proposition 1 when combined with Theorem 4.1 of [16] is the following theorem.

Theorem 1: Let $\left(x^{*}, \gamma^{*}\right)$ denote the optimal solution of the scenario program $P_{S C}^{\prime}$. Under Assumptions 1 and 2 we have that

$$
\begin{aligned}
& \mathbb{P}^{M}\left\{\left\{\theta_{m}\right\}_{m \in \mathscr{M}} \in \Theta^{M}:\right. \\
& \left.\qquad \mathbb{P}\left(\theta \in \Theta: h\left(x^{*}, \gamma^{*}, \theta\right)>0\right)>\varepsilon\right\} \leq \beta, \\
& \text { where } \beta=\sum_{j=0}^{n} \varepsilon^{j}(1-\varepsilon)^{M-j} .
\end{aligned}
$$

The bound obtained from Theorem 1 constitutes a major improvement for this class of problems, since, irrespective of the number of agents $N$, the same number of samples $M$ is required to provide identical robustness certificates for a given decision vector size of $n$. The proof of this theorem, is a direct application of the scenario approach theory (see 
Theorem 2.4 in [19], and Theorem 4.1 in [16], where the number of support support constraints is replaced by the obtained bound for the support rank, namely, $n+1)$. Note that in the absence of Proposition 1, direct application of the scenario approach theory [19] to the problem under consideration would result in (9), however, (10) would be replaced by

$$
\beta=\sum_{j=0}^{n N} \varepsilon^{j}(1-\varepsilon)^{M-j} .
$$

The following corollary connects our results with the initial problem under study $P$. It shows that the guarantees that the optimal solution of problem $P^{\prime}$ will satisfy a new yet unseen constraint of the form (2), corresponding to $\theta \in \Theta$. In other words, we can quantify the probability that the optimal value of the cost function of the program $P$ will deteriorate when a new sample is encountered. With a small abuse of notation let $J(x)=J\left(x,\left\{\left\{\theta_{m}\right\}_{m \in \mathscr{M}}\right)\right.$ be the cost function of the corresponding scenario program of program $P$ and $J^{+}(x)=J\left(x,\left\{\left\{\theta_{m}\right\}_{m \in \mathscr{M}} \cup\{\theta\}\right)\right.$ be the cost function defined over $M+1$ scenarios by taking into account the new sample $\theta$.

Corollary 1: Let $x^{*}$ denote the optimal solution of program $P$. Under Assumptions 1 and 2 we have that

$$
\begin{gathered}
\mathbb{P}^{M}\left\{\left\{\theta_{m}\right\}_{m \in \mathscr{M}} \in \Theta^{M}:\right. \\
\left.\qquad \mathbb{P}\left(\theta \in \Theta: J^{+}\left(x^{*}\right)>J\left(x^{*}\right)\right)>\varepsilon\right\} \leq \beta, \\
\text { where } \beta=\sum_{j=0}^{n} \varepsilon^{j}(1-\varepsilon)^{M-j} .
\end{gathered}
$$

Proof: Let $\left(x^{*}, \gamma^{*}\right)$ be the optimal solution of program $P^{\prime}$, which implies that $\gamma^{*}=\max _{m \in \mathscr{M}} g\left(x^{*}, \theta_{m}\right)$. As such,

$$
\begin{aligned}
& \mathbb{P}\left(\theta \in \Theta: h\left(x^{*}, \gamma^{*}, \theta\right)>0\right)=\mathbb{P}\left(\theta \in \Theta: g\left(x^{*}, \theta\right)>\gamma^{*}\right)= \\
& \mathbb{P}\left(\theta \in \Theta: g\left(x^{*}, \theta\right)>\max _{m \in \mathscr{M}} g\left(x^{*}, \theta_{m}\right)\right)= \\
& \mathbb{P}\left(\theta \in \Theta: \max \left\{g\left(x^{*}, \theta\right), \max _{m \in \mathscr{M}} g\left(x^{*}, \theta_{m}\right)\right\}>\max _{m \in \mathscr{M}} g\left(x^{*}, \theta_{m}\right)\right)= \\
& \mathbb{P}\left(\theta \in \Theta: J^{+}\left(x^{*}\right)>J\left(x^{*}\right)\right),
\end{aligned}
$$

where the second equality follows from the fact that $\gamma^{*}=$ $\max _{m \in \mathscr{M}} g\left(x^{*}, \theta_{m}\right)$, and the last one from the definitions of $J$ and $J^{+}$. Direct substitution of (14) in (9) of Theorem 1, concludes then the proof.

\section{NUMERICAL RESULTS}

We apply our results to the electric vehicle charging problem, introduced in Subsection II-B. Approximating problem $P_{E V}^{\prime}$ by sampling, gives rise to its corresponding scenario program

$$
\begin{gathered}
P_{E V}^{s c}: \min _{(x, \gamma) \in \mathbb{R}^{n N+1}} f(x)+\gamma \\
\text { subject to } x_{i} \in\left[\underline{x}_{i}, \bar{x}_{i}\right], \sum_{t=1}^{n} x_{i}^{(n)} \geq E_{i}, \text { for all } i \in \mathscr{N}, \\
g\left(x, \theta_{m}\right) \leq \gamma, \text { for all } m \in \mathscr{M}
\end{gathered}
$$

Each $A\left(\theta_{m}\right) \in \mathbb{R}^{n \times n}$, is considered to be a diagonal matrix, whose elements $\left\{a\left(\theta_{m}\right)\right\}, m \in \mathscr{M}$ are extracted according to a lognormal distribution, normalised with respect to the number of agents. The elements of $b\left(\theta_{m}\right) \in \mathbb{R}^{n}$ follow a uniform distribution, as in [21]. For each agent $i \in \mathscr{N}$, the upper bound $\bar{x}_{i}$ takes a random integer value in the set $[15,25]$, the lower bound $\underline{x}_{i}$ is set to zero and the final energy to be achieved by the end of the charging cycle is chosen as reported in [21]. The diagonal entries of $A_{0}=$ $\operatorname{diag}\left(\left\{a_{t}\right\}_{t=1}^{n}\right) \in \mathbb{R}^{n \times n}$ and $b_{0}$ are derived by rescaling a winter weekday demand profile in the UK. The scenario program $P_{E V}^{s c}$ is solved in a centralised manner. It is important to stress that our results are not restricted only to centralised versions of optimization algorithms and can in fact be used alongside any optimization algorithm irrespective of its nature, i.e., centralised, decentralised or distributed. We fix the number of time-steps to $n=12$, and solve the scenario program for different values of the number of agents $N \in\{2,4, \ldots, 20\}$. The number of samples we use for each problem is $M=500$. By fixing $\beta=10^{-6}$ and using the bound

$$
\varepsilon=\frac{2}{M}\left[\ln \frac{1}{\beta}+n \ln 2\right],
$$

which is a sufficient condition (see [23]) for satisfaction of (10), we obtain the theoretical violation level $\varepsilon=0.0885$. Note that the dimension we use to provide probabilistic guarantees for the optimal solution is set, in accordance to Theorem 1 to $n+1$ instead of $n N+1$, which circumvents the computational issues related to the rapid surge in dimension due to the multiplication of the number of agents with the number of time slots.

By drawing a different multi-sample for each choice of the number of agents $N=\{2,4, \ldots, 20\}$, we solve the corresponding scenario program for a fixed number of time slots $n=12$. We then repeat this process 25 times (note that the multi-sample used for each repetition is also different) and compute the empirical probability of violation of the obtained optimal solutions, using $M_{\text {test }}=100000$ test samples each time. The mean empirical probability of violation is depicted in Figure 1 in comparison with the theoretical violation level $\varepsilon$. The empirical values are always below the theoretical level of violation, which is constant with the number of agents due to the agent independent nature of our Theorem 1. In addition, the trend in Figure 1 shows, as expected by Theorem 1, that number of agents does not affect the empirical probability of violation.

This result highlights the fact that, given a specified charging duration, the number of samples $M$ required to provide identical probabilistic guarantees, as the size of the fleet of electric vehicles increases, remains constant. This is illustrated in Figure 2, where we show the number of samples required using the results of Theorem 1 versus the number of samples needed to provide the same robustness certificates using the classic results in scenario approach for a different number of agents $N=2, \ldots, 20$. Solid lines correspond to Theorem 1, while dashed-dotted lines correspond to (11). We consider two different cases for the duration of the charging cycle, namely, $n=12$ and $n=24$ hours. 


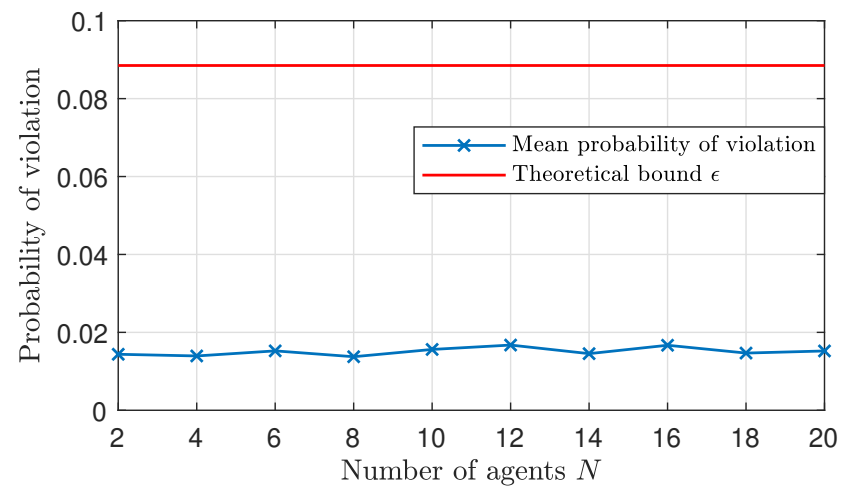

Fig. 1. Mean empirical probability of violation of the optimal solution with respect to the number of agents versus the theoretical violation level $\varepsilon=0.0885$. The number of samples used is $M=500$ and the confidence level is $\beta=10^{-6}$. By drawing a different multi-sample for each choice of the number of agents $N=\{2,4, \ldots, 20\}$, we solve the corresponding scenario program for a fixed number of time slots $n=12$. We then repeat this process 25 times (note that the multi-sample used for each repetition is also different) and compute the empirical probability of violation of the obtained optimal solutions, using $M_{\text {test }}=100000$ test samples.

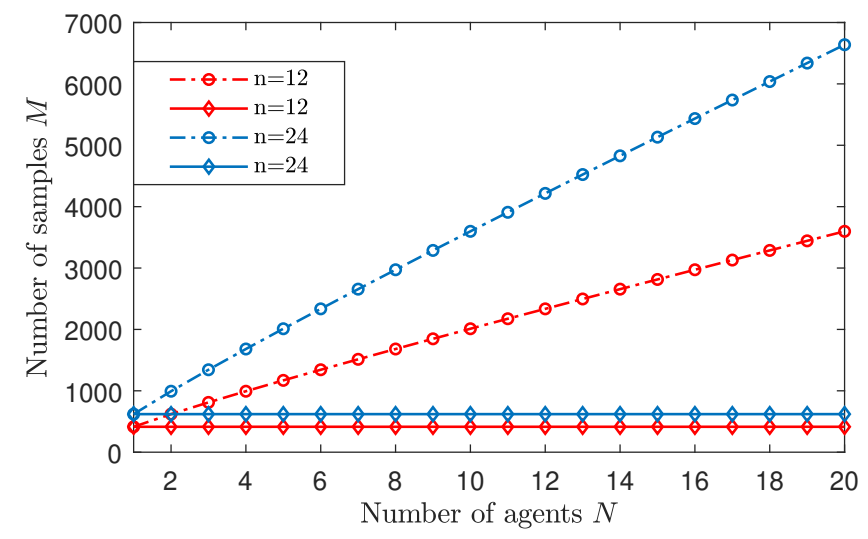

Fig. 2. The number of samples required with respect to the number of agents $N=2, \ldots, 20$ using the results of Theorem 1 versus the one that would have been obtained if (11) is used instead. Solid lines correspond to Theorem 1, while dashed-dotted lines correspond to (11). We consider to different cases for the duration of the charging cycle, namely, $n=12$ and $n=24$ hours.

\section{CONCLUDing REMARKS}

Focusing on a specific class of optimization programs, we provided agent independent probabilistic guarantees for the optimal solution in an a priori fashion. The importance of this result is better shown in the context of the EV-charging control problem, where significantly improved robustness certificates are obtained for the optimal solution returned by any convex optimization algorithm. The numerical study supports our results as it shows that, irrespective of the number of agents, the same number of samples are required to offer probabilistic feasibility guarantees of the same quality.

Effort is being made towards extending our results to provide agent independent probabilistic guarantees for Nash equilibria of a certain class of games affected by uncertainty, as well as on investigating a more general case, where each agent participating in the game has a different uncertain function in her cost.

\section{REFERENCES}

[1] D. Ioli, L. Deori, A. Falsone, and M. Prandini, "A two-layer decentralized approach to the optimal energy management of a building district with a shared thermal storage," IFAC-PapersOnLine, vol. 50, pp. 8844-8849, 072017.

[2] B. Gharesifard, T. Başar, and A. Domínguez-García, "Price-based coordinated aggregation of networked distributed energy resources," IEEE Transactions on Automatic Control, vol. 61, no. 10, pp. 29362946, 12016.

[3] D. Paccagnan, B. Gentile, F. Parise, M. Kamgarpour, and J. Lygeros, "Nash and wardrop equilibria in aggregative games with coupling constraints," IEEE Transactions on Automatic Control, vol. 64, no. 4, pp. 1373-1388, April 2019.

[4] D. Acemoglu and M. K. Jensen, "Aggregate comparative statics," Games and Economic Behavior, vol. 81, pp. 27 - 49, 2013. [Online]. Available: http://www.sciencedirect.com/science/article/pii/ S0899825613000523

[5] J. Ghaderi and R. Srikant, "Opinion dynamics in social networks with stubborn agents: Equilibrium and convergence rate," Automatica, vol. 50, no. 12, pp. 3209 - 3215, 2014. [Online]. Available: http://www.sciencedirect.com/science/article/pii/S0005109814004154

[6] D. Acemoglu and A. Ozdaglar, "Opinion dynamics and learning in social networks," Dynamic Games and Applications, vol. 1, no. 1, pp. 3-49, 2011.

[7] D. Bertsekas and J. Tsitsiklis, "Parallel and distributed computation : numerical methods / dimitri p. bertsekas, john n. tsitsiklis," Athena Scientific, 011989.

[8] N. Parikh and S. Boyd, "Proximal algorithms," Foundations and

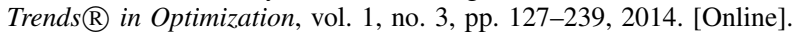
Available: http://dx.doi.org/10.1561/2400000003

[9] L. Deori, K. Margellos, and M. Prandini, "Regularized jacobi iteration for decentralized convex quadratic optimization with separable constraints," IEEE Transactions on Control Systems Technology, vol. 27, pp. 1636-1644, 2016.

[10] S. Grammatico, "Aggregative control of competitive agents with coupled quadratic costs and shared constraints," in 2016 IEEE 55th Conference on Decision and Control (CDC), Dec 2016, pp. 35973602.

[11] _ - "Dynamic control of agents playing aggregative games with coupling constraints," IEEE Transactions on Automatic Control, vol. 62, no. 9, pp. 4537-4548, 2017.

[12] S. Grammatico, F. Parise, M. Colombino, and J. Lygeros, "Decentralized convergence to nash equilibria in constrained deterministic mean field control," IEEE Transactions on Automatic Control, vol. 61, no. 11 , pp. 3315-3329, 2016.

[13] M. C. Campi and G. C. Calafiore, "The scenario approach to robust control design," IEEE Transactions on automatic control, vol. 51, no. 5, pp. 742-753, 2006.

[14] M. C. Campi and S. Garatti, "The exact feasibility of randomized solutions of uncertain convex programs," SIAM J. on Optimization, vol. 19, no. 3, pp. 1211-1230, Nov. 2008. [Online]. Available: http://dx.doi.org/10.1137/07069821X

[15] M. C. Campi, S. Garatti, and M. Prandini, "The scenario approach for systems and control design," Annual Reviews in Control, vol. 33, pp. 149-157, 2008.

[16] G. Schildbach, L. Fagiano, and M. Morari, "Randomized solutions to convex programs with multiple chance constraints," SIAM Journal on Optimization, vol. 23, 052012.

[17] D. Bai, T. Carpenter, and J. Mulvey, "Making a case for robust optimization models," Management Science, vol. 43, pp. 895-907, 07 1997.

[18] J. R. Birge and F. Louveaux, Introduction to Stochastic Programming. New York, NY, USA: Springer-Verlag, 1997.

[19] M. C. Campi and S. Garatti, "The exact feasibility of randomized solutions of uncertain convex programs," SIAM Journal on Optimization, vol. 19, no. 3, pp. 1211-1230, 2008.

[20] F. Fele and K. Margellos, "Probabilistic sensitivity of nash equilibria in multi-agent games: a wait-and-judge approach," in 2019 IEEE 58th Conference on Decision and Control (CDC), Dec 2019, pp. 50265031. 
[21] F. Fele and K. Margellos, "Probably approximately correct nash equilibrium learning," IEEE Transactions on Automatic Control, 2019, under review, 2019.

[22] S. Boyd and L. Vandenberghe, Convex Optimization. USA: Cambridge University Press, 2004.

[23] M. Campi and S. Garatti, Introduction to the Scenario Approach, 11 2018. 